\title{
PTERIDÓFITAS DE UMA ÁREA REMANESCENTE DE FLORESTA ATLÂNTICA DO ESTADO DE PERNAMBUCO, BRASIL ${ }^{1}$
}

\author{
Sandra Tereza Ambrósio ${ }^{2}$ \\ Iva Carneiro Leão Barros
}

Recebido em 28/5/97. Aceito em 22/9/97

\begin{abstract}
RESUMO - (Pteridófitas de uma área remanescente de Floresta Atlântica do Estado de Pernambuco, Brasil). É apresentado um "checklist" das pteridófitas ocorrentes na Reserva Ecológica de Jangadinha, Município de Jaboatão dos Guararapes, como contribuição aos estudos da pteridoflora de áreas de Floresta Atlântica do Estado de Pernambuco. Informações sobre a auto-ecologia das espécies também são apresentadas. Coletas foram realizadas em cinco pontos, denominados de Banho-Frio - I, Banho-Frio II, Área do Reservatório, Área do Açude e Mata do Curado. Foram reconhecidos 25 táxons distribuidos em 14 familias: Schizaeaceae ( 2 spp.), Gleicheniaceae (2 spp.), Hymenophyllaceae (1 sp.), Cyatheaceae (1 sp.), Pteridaceae (7 spp.), Vittariaceae (1 sp.), Dennstaedtiaceae (2 spp.), Thelypteridaceae (2 spp.), Dryopteridaceae (1 sp.), Davalliaceae (1 sp.), Blechnaceae (1 sp.), Polypodiaceae (2 spp.), Lycopodiaceae (1 sp.) e Selaginellaceae (1 sp.). Em todos os pontos de coletas há pteridófitas, sendo que Banho-Frio I é o mais rico e a Mata do Curado relativamente pobre em número de espécies. A maior diversidade florística foi encontrada nos micro-habitats de interior e margem das matas, principalmente em barrancos úmidos.
\end{abstract}

Palavras-chave: pteridófitas, Floresta Atlântica, Reserva Ecológica de Jangadinha

\begin{abstract}
Floristic survey of the pteridophytes from a remnant area of Atlantic Forest, Pernambuco State, Brazil). A checklist of the pteridophytes occurrence in Ecological Reserve of Jangadinha, in the municipality of Jaboatão dos Guararapes was made as a contribution to the study of remnant area of Atlantic Forest of Pernambuco, Brazil. Auto-ecology information about the species are included. Collections were made in five points of the Reserve, namely Banho-Frio - I, Banho-Frio - II, Área do Açude, Área do Reservatório and Mata do Curado. Twenty-five species of the following 14 families were recognized: Schizaeaceae ( 2 spp.), Gleicheniaceae ( 2 spp.), Hymenophyllaceae (1 sp.), Cyatheaceae (1 sp.), Pteridaceae ( 7 spp.), Vittariaceae (1 sp.), Dennstaedtiaceae (2 spp.), Thelypteridaceae (2 spp.), Dryopteridaceae (1 sp.), Davalliaceae (1sp.), Blechnaceae (1 sp.), Polypodiaceae (2 spp.), Lycopodiaceae (1 sp.) and Selaginellaceae (1 sp.). Pteridophytes occurred in all points studied. Banho-Frio-I was richer in species than the other places, and Mata do Curado is relatively poor. The microhabitat with most species were those enclosed inside the forest and their edge, mainly in the humid slopes.
\end{abstract}

Key words: pteridophytes, Atlantic Forest, Ecological Reserve of Jangadinha

\footnotetext{
Parte da Dissertação de Mestrado da primeira autora

Bolsista, CNPq (DCR), E-mail: sandra@cpatsa.embrapa.br

Centro de Ciências Biológicas, Universidade Federal de Pernambuco, CEP 50670-710, Recife, PE
} 


\section{Introdução}

Na região Nordeste do Brasil, de modo geral, as pteridófitas têm uma distribuição relativamente ampla, ocorrendo nas diversas zonas fitogeográficas que abrangem desde a Serra do Baturité - CE (Brade 1940; Braga 1951) até a Chapada Diamantina - BA (Prado 1995), além daquelas ocorrentes em áreas de caatinga (Barros et al. 1989b).

No Estado de Pernambuco, estes vegetais ocorrem mais freqüentemente em áreas de Floresta Atlântica. No que se refere a essas áreas, estudos vêm sendo desenvolvidos principalmente por Barros et al. (1988), Barros et al. (1989a) e Farias et al. (1992), que registraram a ocorrência de aproximadamente 270 espécies de pteridófitas.

No entanto, algumas áreas do Estado de Pernambuco, como é o caso da Reserva Ecológica de Jangadinha, ainda estão por merecer uma exploração florística mais detalhada no que se refere às pteridófitas, por serem áreas de Floresta Atlântica e ainda relativamente preservadas.

Desta forma, o presente estudo é uma contribuição ao conhecimento da composição florística de áreas remanescentes de Floresta Atlântica ocorrentes no Nordeste brasileiro, particularmente no Estado de Pernambuco.

\section{Material e métodos}

O Estado de Pernambuco ocupa uma área de $98.079 \mathrm{~km}^{2}$ dentro da região Nordeste e sua vegetação encontra-se tipicamente distribuída em três diferentes zonas fitogeográficas sendo Zona da Mata, Agreste e Sertão. A Zona da Mata ou Floresta Atlântica caracteriza-se por um número significativo de espécies vegetais e ocupa aproximadamente 15,7\% da área total do Estado de Pernambuco (Andrade-Lima 1960; Rizzini et al. 1988).

A Reserva Ecológica de Jangadinha (Lat. $8^{\circ} 05^{\prime} \mathrm{S}$, Long. $34^{\circ} 58^{\prime} \mathrm{O}$ ) está situada numa área remanescente de Floresta Atlântica, ocupando 84,68 ha (cerca de 0,34\%) do Município de Jaboatão dos Guararapes, PE, a aproximadamente $25 \mathrm{~km}$ da cidade do Recife. A área é caracterizada por alguns autores como Floresta Estacional Perenifolia Costeira e nela se encontra o Açude de Jangadinha, que compreende uma área bem significativa dentro da Reserva e está encarregado, entre outros, do abastecimento de água de parte da cidade do Recife (Andrade-Lima 1960; Fidem 1987).

A área da Reserva, embora apresente problemas de devastações em conseqüência de invasões antrópicas, o que vem ocasionando o desaparecimento de várias espécies, possui locais relativamente preservados, onde ainda não foram realizados trabalhos de cunho florístico no que se refere às pteridófitas.

Assim, realizaram-se coletas em cinco pontos da Reserva: Banho Frio - I, Banho Frio - II, Área do Reservatório, Área do Açude, Mata do Curado.

As espécies de pteridófitas incluídas neste trabalho foram identificadas com base nas chaves analíticas contidas principalmente nos trabalhos de Kramer (1957), Stolze (1976), Alston et al. (1981), Tryon \& Tryon (1982), Proctor (1985), Mickel \& Beitel (1988), Tryon \& Stolze $(1989,1991,1992)$ e Lellinger (1991). O sistema de classificação adotado para a seqüência de apresentação dos táxons, foi o proposto por 
Tryon \& Tryon (1982). As informações complementares sobre a auto-ecologia das espécies baseiam-se nos conceitos apresentados por Daubenmire (1974).

Após identificação, o material foi depositado no Herbário UFP (Universidade Federal de Pernambuco).

Resultados e discussão

"Checklist" das pteridófitas ocorrentes na Reserva Ecológica de Jangadinha:

$$
\begin{aligned}
& \text { PTERIDOPHYTA } \\
& \text { BLECHNACEAE }
\end{aligned}
$$

Blechnum serrulatum Rich.

Col.: Ambrósio, Germano \& Valdevino 23

CYATHEACEAE

Trichipteris microdonta (Desv.) R.M. Tryon

Col.: Ambrósio \& Barros s.n; Ambrósio 34

DAVALLIACEAE

Nephrolepis biserrata (Sw.) Schott

Col.: Ambrósio \& Barros 24; Ambrósio s.n; Ambrósio 27

DENNSTAEDTIACEAE

Lindsaea cf. ovoidea Fée

Col.: Ambrósio 21

Lindsaea quadrangularis Raddi

Col.: Ambrósio \& Barros 22

DRYOPTERIDACEAE

Triplophyllum cf. funestum (Kunze) Holttum

Col.: Ambrósio 47

GLEICHENIACEAE

Dicranopteris flexuosa (Schrad.) Underw.

Col.: Ambrósio 8

Dicranopteris pecinata (Willd.) Underw.

Col.: Ambrósio \& Barros 7; Ambrósio 26

HYMENOPHYLLACEAE

Trichomanes pinnatum Hedw.

Col.: Ambrósio \& Barros 16

LYCOPODIACEAE

Lycopodiella cernua (L.) Pic.Serm.

Col.: Ambrósio 48; Ambrósio s.n

$$
\text { POLYPODIACEAE }
$$

Polypodium triseriale Sw. var. triseriale

Col.: Ambrósio \& Barros s.n; Ambrósio s.n

Microgramma vacciniifolia (Langsd. \& Fisch.) Copel.

Col.: Ambrósio \& Barros 1

$$
\text { PTERIDACEAE }
$$

Pityrogramma calomelanos (L.) Link. var. calomelanos 
Col.: Ambrósio s.n; Ambrósio 42

Adiantum dolosum Kunze

Col.: Ambrósio \& Barros 4; Ambrósio 19

Adiantum latifolium Lam.

Col.: Ambrósio 15; Ambrósio \& Barros 38

Adiantum obliquum Willd.

Col.: Ambrósio \& Barros 3; Ambrósio 12; Ambrósio s.n

Adiantum petiolatum Desv.

Col.: Ambrósio s.n

Adiantum terminatum Kunze ex Miq.

Col.: Ambrósio \& Yano 25; Ambrósio \& Barros 18

Pteris vittata $\mathrm{L}$.

Col.: Ambrósio \& Barros 5

SCHIZAECEAE

Lygodium venustum $\mathrm{Sw}$.

Col.: Ambrósio 14

Lygodium volubile $\mathrm{Sw}$.

Col.: Ambrósio s.n; Ambrósio 11

SELAGINELLACEAE

Selaginella muscosa Spring

Col.: Ambrósio \& Barros 17

THELYPTERIDACEAE

Thelypteris dentata (Forssk.) E. P. St. John

Col.: Ambrósio \& Barros s.n; Ambrósio 32; Ambrósio 50

Thelypteris serrata (Cav.) Alston

Col.: Ambrósio \& Barros s.n; Ambrósio 29

VITTARIACEAE

Vittaria lineata $(\mathrm{L}.) \mathrm{Sm}$.

Col.: Ambrósio \& Barros 6

De modo geral, as floras mais diversificadas no Nordeste brasileiro, sobretudo aquelas do Estado de Pernambuco, ocorrem em ambientes onde há umidade no solo em decorrência da presença de açudes, rios e riachos (Brade 1940; Barros et al. 1989a,b), o que é confirmado no presente estudo.

Fazendo-se uma analogia entre a flora da Reserva Ecológica de Jangadinha e a flora de uma área de Floresta Tropical Úmida no Zâmbia, estudada por Kornás (1979), no centro-oeste da África, constata-se que são comuns aos dois ambientes os seguintes gêneros considerados higrófilos: Adiantum, Pteris, Trichomanes, Triplophyllum, Vittaria, Blechnum, Nephrolepis, Thelypteris e Selaginella. Também ocorrem nas duas áreas os seguintes gêneros ditos xerófilos: Lygodium, Dicranopteris e Lycopodiella. Quanto à riqueza específica do grupo, sabe-se que a família Pteridaceae é de grande representatividade e importância nos neotrópicos (Tryon \& Tryon 1982). $\mathrm{Na}$ Reserva estudada a família melhor representada também é Pteridaceae, com $28 \%$ do total de espécies, seguida de Dennstaedtiaceae, Schizaeaceae, Gleicheniaceae, 
Thelypteridaceae e Polypodiaceae com $8 \%$ cada, enquanto que as Hymenophyllaceae, Cyatheaceae, Vittariaceae, Davalliaceae, Blechnaceae, Dryopteridaceae, Lycopodiaceae e Selaginellaceae representam $4 \%$ cada uma (Fig. 1). Estes dados também vêm a confirmar aqueles obtidos por Barros et al. (1988) que comentam que no Estado de Pernambuco a família Pteridaceae está bem representada.

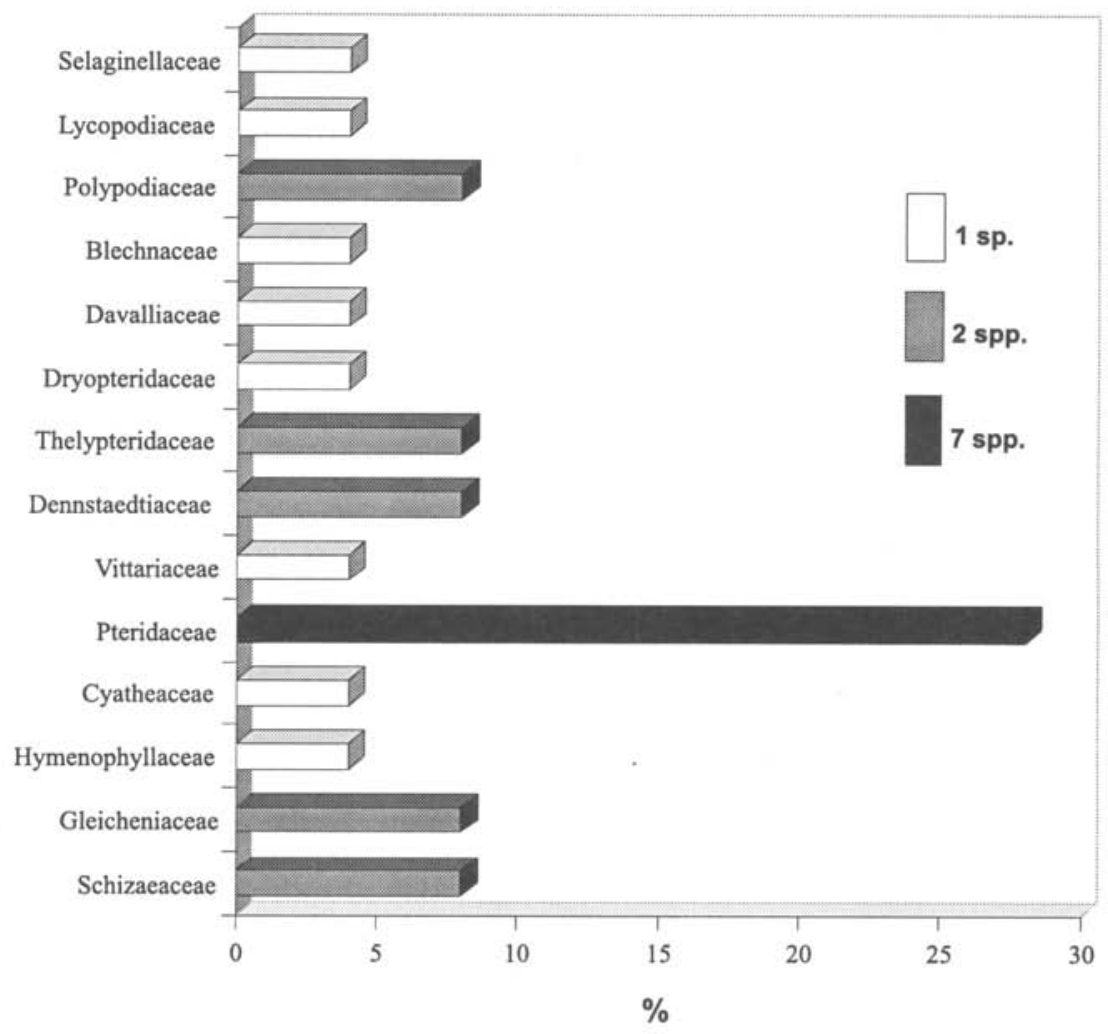

Figura 1: Frequência percentual (\%) das pteridófitas da Reserva Ecológica de Jangadinha, Jaboatão dos Guararapes, PE.

Adiantum foi o gênero mais representativo com cinco espécies: Adiantum dolosum, A. latifolium, A. obliquum, A. petiolatum e A. terminatum. De acordo com Lellinger (1991), problemas taxonômicos vêm sendo encontrados na distinção de espécies de Adiantum, algumas inclusive híbridas. De fato, a maioria dessas espécies foram de difícil identificação, algumas por falta de material fértil, outras por apresentarem características taxonômicas muito próximas.

No que se refere à ocorrência de espécies por pontos de coleta, de um modo geral, as pteridófitas estão amplamente distribuídas em todos os pontos, com exceção da Mata do Curado onde ocorrem poucos representantes. Os pontos de maior riqueza são Banho-Frio-I e Açude, onde ocorrem 21 e 16 espécies, repectivamente (Tab. 1). 
Tabela 1. Distribuição das pteridófitas na Reserva Ecológica de Jangadinha, Jaboatão dos Guararapes, PE, Brasil.

\begin{tabular}{lccccc}
\hline & \multicolumn{5}{c}{ Pontos de coletas } \\
\hline Espécies & Banho & Banho & Area & Area do & Mata do \\
& Frio I & Frio II & Reservatório & Açude & Curado \\
\hline
\end{tabular}

Blechnaceae

Blechnum serrulatum

Cyatheaceae

Trichipteris microdonta

Davalliaceae

Nephrolepis biserrata

Dennstaedtiaceae

Lindsaea $\mathrm{cf}$. ovoidea

Lindsaea quadrangularis

Dryopteridaceae

Triplophyllum cf. funestum

Gleicheniaceae

Dicranopteris flexuosa

Dicranopteris pectinata

Hymenophyllaceae

Trichomanes pinnatum

Lycopodiaceaea

Lycopodiella cernua

Polypodiaceae

Microgramma vacciniifolia

Polypodium triseriale var. triseriale

Pteridaceae

Adiantum dolosum

Adiantum latifolium

Adiantum obliquum

Adiantum petiolatum

Adiantum terminatum

Pityrogramma calomelanos

var. calomelanos

Pteris vittata

Schizaeaceae

Lygodium venustum

Lygodium volubile

Selaginellaceae

Selaginella muscosa

Thelypteridaceae

Thelypteris dentata

Thelypteris serrata

Vittariaceae

Vittaria lineata

Convenção: + presente/-ausente

Com relação aos micro-habitats, destacam-se os ambientes sombreados, no interior e nos barrancos úmidos (bordos de mata), onde ocorrem 16 espécies (Tab. 2). De acordo com Ranal (1995), a maioria das espécies de pteridófitas ocorrem em solos que retêm maior quantidade de água, mantendo seu rizoma e raízes na camada superficial do substrato, onde há maior umidade em relação às partes mais profundas. 
Tabela 2. Distribuição das pteridófitas nos micro-habitats ocorrentes na Reserva Ecológica de Jangadinha, Jaboatão dos Guararapes, PE, Brasil.

\begin{tabular}{|c|c|c|c|c|c|}
\hline \multirow{3}{*}{ Espécies } & \multicolumn{5}{|c|}{ Micro-habitats } \\
\hline & \multicolumn{2}{|c|}{ Interior da mata } & \multirow{2}{*}{$\begin{array}{l}\text { Bordos } \\
\text { da mata } \\
\text { (barrancos) }\end{array}$} & \multirow{2}{*}{$\begin{array}{l}\text { Próximo } \\
\text { ao Açude }\end{array}$} & \multirow{2}{*}{$\begin{array}{l}\text { Area } \\
\text { periodica- } \\
\text { mente } \\
\text { encharcada }\end{array}$} \\
\hline & clareira & $\begin{array}{l}\text { área encoberta } \\
\text { por vegetação }\end{array}$ & & & \\
\hline \multicolumn{6}{|l|}{ Blechnaceae } \\
\hline \multicolumn{6}{|l|}{ Cyatheaceae } \\
\hline Trichipteris microdonta & - & - & + & - & + \\
\hline \multicolumn{6}{|l|}{ Davalliaceae } \\
\hline $\begin{array}{l}\text { Nephrolepis biserrata } \\
\text { Dennstaedtiaceae }\end{array}$ & - & + & + & + & + \\
\hline \multicolumn{6}{|l|}{ Dennstaedtiaceae } \\
\hline $\begin{array}{l}\text { Lindsaea cf. ovoidea } \\
\text { Lindsaea quadrangularis }\end{array}$ & - & + & - & - & - \\
\hline \multicolumn{6}{|l|}{ Dryopteridaceae } \\
\hline Triplophyllum cf. funestum & - & - & + & - & - \\
\hline \multicolumn{6}{|l|}{ Gleicheniaceae } \\
\hline Dicranopteris flexuosa & - & - & + & + & - \\
\hline Dicranopteris pectinata & - & - & + & + & - \\
\hline \multicolumn{6}{|l|}{ Hymenophyllaceae } \\
\hline Trichomanes pinnatum & - & + & - & - & - \\
\hline \multicolumn{6}{|l|}{ Lycopodiaceaea } \\
\hline Lycopodiella cernua & - & - & + & - & - \\
\hline \multicolumn{6}{|l|}{ Polypodiaceae } \\
\hline Microgramma vacciniifolia & - & + & + & + & - \\
\hline Polypodium triseriale var. triseriale & + & - & + & + & + \\
\hline \multicolumn{6}{|l|}{ Pteridaceae } \\
\hline Adiantum dolosum & - & + & - & - & - \\
\hline Adiantum latifolium & - & + & - & - & - \\
\hline Adiantum obliquum & - & + & - & & - \\
\hline Adiantum petiolatum & - & + & - & - & - \\
\hline Adiantum terminatum & - & + & + & - & - \\
\hline Pityrogramma calomelanos var. calomelanos & $s-$ & + & + & + & - \\
\hline Pteris vittata & - & - & + & - & - \\
\hline \multicolumn{6}{|l|}{ Schizaeaceae } \\
\hline Lygodium venustum & + & - & + & - & - \\
\hline Lygodium volubile & + & + & + & - & - \\
\hline \multicolumn{6}{|l|}{ Selaginellaceae } \\
\hline Selaginella muscosa & - & - & + & - & - \\
\hline \multicolumn{6}{|l|}{ Thelypteridaceae } \\
\hline Thelypteris dentata & - & - & + & + & + \\
\hline Thelypteris serrata & - & - & - & + & + \\
\hline \multicolumn{6}{|l|}{ Vittariaceae } \\
\hline Vittaria lineata & - & + & - & - & - \\
\hline
\end{tabular}

De fato, como citado acima, grande número de espécies de pteridófitas ocorrentes na Reserva Ecológica de Jangadinha, localizam-se em áreas úmidas ou relativamente úmidas, que correspondem ao Banho-Frio-I e Açude. Ainda com relação a esses tipos de ambientes, vale salientar que Trichomanes pinnatum ocorre exclusivamente em 
locais úmidos e sombreados, assim como é comentado por Windisch (1983) para a Serra Ricardo Franco, embora esta não seja área de Floresta Atlântica.

Quanto ao hábito, foram constatadas três formas no presente estudo: plantas terrestres, epifitas e escandentes, em ordem decrescente de representatividade. Quanto às epífitas, nota-se que estas ocorrem preferencialmente sobre visgueiro (Parkia pendula Benth.) e dendê (Elaies guineensis Jack). Foram observadas algumas epífitas também sobre troncos mortos de algumas dicotiledôneas.

Os resultados obtidos neste trabalho são relativamente significativos, principalmente pelo fato deste ser pioneiro na Reserva Ecológica de Jangadinha, aliado ao fato da mesma ser uma das poucas áreas remanescentes de Floresta Atlântica ocorrentes no Estado de Pernambuco. Acredita-se que a referida flora provavelmente pode ter sido mais diversificada anteriormente, quando a área não apresentava alterações antrópicas. Desta forma, sugere-se maiores cuidados em relação a estas áreas de Florestas como a Reserva Ecológica de Jangadinha, em termos de preservação da vegetação, pelo que representam no ecossistema, como remanescentes de Floresta Atlântica, bem como na manutenção de nascentes.

\section{Agradecimentos}

Os autores agradecem ao Dr. Jefferson Prado (Instituto de Botânica de São Paulo) pelas sugestões e críticas apresentadas e ao CNPq pelo apoio financeiro.

\section{Referências bibliográficas}

Alston, A.H.G.; Jermy, A. C. \& Rankin, J. M. 1981. The genus Selaginella in Tropical South America. Bulletin of the British Museum (Natural History) Botany Series 9(4): 233-330.

Andrade-Lima, D. 1960. Estudos Fitogeográficos de Pernambuco. Instituto de Pesquisas Agronômicas. Recife. 5: 305-341.

Barros, I.C.L.; Lira, O.C. \& Silva, A. de J.R. 1988. Distribuição geográfica das Pteridófitas ocorrentes no Estado de Pernambuco, Brasil. Acta Botanica Brasilica 2(1-2): 47-86.

Barros, I.C.L.; Silva, A.J.R. \& Silva, L.L.S. 1989a. Levantamento florístico das pteridófitas ocorrentes na zona das Caatingas do Estado de Pernambuco. Biologica Brasilica 1(2): 143-159.

Barros, I.C.L.; Silva, A.R.J. \& Costa, M.C.D. 1989b. Adições à Flora Pteridofítica do Estado de Pernambuco. Biologica Brasilica. 1(1): 79-83.

Brade, A. C. 1940. Contribuição para o estudo da flora Pteridophyta da serra do Baturité- Estado do Ceará. Rodriguésia 1(13): 289-314.

Braga, R. 1951. Pteridófitas do Ceará. Instituto do Ceará, Fortaleza.

Daubenmire, R.F. 1974. Plants and environment: A textbook of plant autecology. Washington State University, John Wiley \& Sons.

Farias, M.C.A; Belo, M.A.M. \& Barros, I.C.L. 1992. Pteridófitas da Reserva de Caetés (Paulista - PE). Broteria 2(65): 147-162.

FIDEM - 1987. Fundação de Desenvolvimento da Região Metropolitana do Recife. Recife. Reservas Ecológicas. Série de Desenvolvimento Urbano e Meio Ambiente.

Kornás, J. 1979. Distribution and Ecology of the Pteridophytes in Zâmbia. Polska Akademia Nauk Wydzial II Nauk Biologicznych.

Kramer, K.U. 1957. The revision of the genus Lindsaea in the New World, with notes on allied genera. Acta Botanica Neerlandica 6: 97-290.

Lellinger, D.B. 1991. Common and confusing bipinnate-dimidiate Adiantums of Tropical America. American Fern Journal 81( 3 ): 99-102.

Mickel J.T. \& Beitel, M.J. 1988. Pteridophyte Flora of Oaxaca. Memoirs of the New York Botanical Garden 46: $1-568$. 
Prado, J. 1995. Ferns. Pp. 79-8, 85-110. In B.L. Stannard. (Ed.), Flora of the Pico das Almas, Chapada Diamantina - Bahia, Brazil. Royal Botanic Gardens, Key.

Proctor, G.R. 1985. Ferns of Jamaica. British Museum (Natural History). London.

Ranal, M.A. 1995. Estabelecimento de pteridófitas em mata mesófila semidecidua do Estado de São Paulo. 3. Fenologia e sobrevivência dos individuos. Revista Brasileira de Biologia 55(4): 777-787.

Rizzini, C.T.; Coimbra-Filho, A.F. \& Houaiss, A. 1988. Ecossistemas Brasileiros. Rio de Janeiro. Index, Enge -Rio.

Stolze, R.G. 1976. Ferns and fern allies of Guatemala. Part I. Ophioglossaceae through Cyatheaceae. Fieldiana Botany 39 (1): 1-130.

Tryon, R.M. \& Stolze, R.G. 1989. Pteridophyta of Peru, Part. II. 13 - Pteridaceae-Dennstaedtiaceae - 17. Fieldiana Botany 22: 1-128.

Tryon, R.M. \& Stolze, R.G. 1991. Pteridophyta of Peru, Part. IV. 17 - Dryopteridaceae. Fieldiana Botany 27 . $1-176$.

Tryon, R.M. \& Stolze, R. G. 1992. Pteridophyta of Peru. Part III. 16 - Thelypteridaceae. Fieldiana Botany 29: $1-80$.

Tryon, R.M. \& Tryon, A.F. 1982. Ferns and allied plants, with special reference to Tropical America. New York. Spring-Verlag.

Windisch, P. G. 1983. Pteridófitas da serra Ricardo Franco (Estado de Mato Grosso) e aspectos dos seus macrohabitats. São José do Rio Preto. Universidade Estadual Paulista “Júlio de Mesquita Filho". Tese de Livre Docência. 